\title{
AN ALGEBRAIC SATO-TATE GROUP AND SATO-TATE CONJECTURE
}

\author{
GRZEGORZ BANASZAK AND KIRAN S. KEDLAYA
}

\begin{abstract}
We make explicit a construction of Serre giving a definition of an algebraic Sato-Tate group associated to an abelian variety over a number field, which is conjecturally linked to the distribution of normalized $L$-factors as in the usual Sato-Tate conjecture for elliptic curves. The connected part of the algebraic Sato-Tate group is closely related to the Mumford-Tate group, but the group of components carries additional arithmetic information. We then check that in many cases where the Mumford-Tate group is completely determined by the endomorphisms of the abelian variety, the algebraic SatoTate group can also be described explicitly in terms of endomorphisms. In particular, we cover all abelian varieties (not necessarily absolutely simple) of dimension at most 3; this result figures prominently in the analysis of SatoTate groups for abelian surfaces given recently by Fité, Kedlaya, Rotger, and Sutherland.
\end{abstract}

\section{INTRODUCTION}

Let $F$ be a number field with absolute Galois group $G_{F}$. Let $A$ be an abelian variety of dimension $g \geq 1$ over $F$. Pick a prime number $l$ and consider the action of $G_{F}$ on the $l$-adic Tate module of $A$. A theorem of Weil implies that for each prime ideal $\mathfrak{p}$ of $F$ at which $A$ has good reduction, the characteristic polynomial $L(A / F, T)$ of any geometric Frobenius element of $G_{F}$ corresponding to $\mathfrak{p}$ is a monic polynomial with integer coefficients, whose roots in the complex numbers all have absolute value $q^{1 / 2}$ for $q$ the absolute norm of $\mathfrak{p}$.

One can ask how the renormalized characteristic polynomials $\bar{L}(A / F, T)=$ $q^{-g} L\left(A / F, q^{1 / 2} T\right)$ are distributed in the limit as $q \rightarrow \infty$. For $A$ an elliptic curve without complex multiplication, the Sato-Tate conjecture predicts equidistribution with respect to the image of the Haar measure on $\mathrm{SU}(2)$; this result is now known when $F$ is a totally real field BLGG], building upon recent advances in the modularity of Galois representations led by R. Taylor. For $A$ an elliptic curve with complex multiplication, the situation is easier to analyze: for all $F$, one can prove equidistribution for the image of the Haar measure on a certain subgroup of $\mathrm{SU}(2)$. This group may be taken to be $\mathrm{SO}(2)$ when $F$ contains the field of complex multiplication; otherwise, one must instead take the normalizer of $\mathrm{SO}(2)$ in $\mathrm{SU}(2)$

With this behavior in mind, it is reasonable to formulate an analogous Sato-Tate conjecture for general $A$, in which the equidistribution is with respect to the image

Key words and phrases. Mumford-Tate group, Algebraic Sato-Tate group.

Thanks to Jeff Achter for the reference to Ch. G. Banaszak was supported by the NCN (National Center for Science of Poland) grant NN 201607440 and UC San Diego during visit Dec. 2010 - June 2011. K.S. Kedlaya was supported by NSF CAREER grant DMS-0545904, NSF grant DMS-1101343, DARPA grant HR0011-09-1-0048, MIT (NEC Fund, Cecil and Ida Green professorship), and UC San Diego (Stefan E. Warschawski professorship). 
of the Haar measure on a suitable compact Lie group. Such a conjecture seems to have been first formulated by Serre [Se4] in the language of motives. More recently, Serre has given an alternate formulation in terms of $l$-adic Galois representations [Se5, §8].

The purpose of this paper is to make explicit one aspect of Serre's $l$-adic construction of the putative Sato-Tate group, namely the role of an algebraic group closely related to (but distinct from) the classical Mumford-Tate group associated to $A$. This algebraic Sato-Tate group has the property that its connected part is conjecturally determined by the Mumford-Tate group; however, whereas the Mumford-Tate group is by construction connected, the algebraic Sato-Tate group has a component group with Galois-theoretic meaning. For example, in those cases where the Mumford-Tate group is determined entirely by the endomorphisms of $A$ (e.g., the cases studied by the first author jointly with W. Gajda and P. Krason in [BGK1, BGK2]), we can determine the algebraic Sato-Tate group and interpret its component group as the Galois group of the minimal extension of $F$ over which all geometric endomorphisms are defined.

One key motivation for this work is to establish the properties of the algebraic Sato-Tate group for all abelian varieties of dimension at most 3. (Note that includes only cases where the Mumford-Tate group is determined by endomorphisms, as the first counterexamples are Mumford's famous examples in dimension 4.) This result is applied by the second author in [?] in order to classify the possible SatoTate groups arising in dimension 2, and makes the corresponding classification in dimension 3 feasible as well.

In cases where the Mumford-Tate group is not determined by endomorphisms, one can still exhibit a natural candidate for the algebraic Sato-Tate group by following Serre's motivic construction [Se4. Based on computations in $\S 7$ and $\S 8$, using the Tannakian formalism, we define in $\S 9$ the algebraic Sato-Tate group $A S T_{K}(A)$ assuming that the Mumford-Tate group is the connected component of the motivic Mumford-Tate group (a hypothesis put forward by Serre in [Se4, sec. 3.4]). Then in $\S 9$ we prove (see Theorem 9.8) that the group $A S T_{K}(A)$ satisfies the conditions (2.4)-(2.6) of Remark (2.5) which one naturally expects to hold for the algebraic Sato-Tate group. If a suitable motivic version of the Mumford-Tate conjecture holds, then we prove that the algebraic Sato-Tate conjecture also holds. We also show that the $\operatorname{AST}_{K}(A)$ group defined in $\S 9$ agrees with the algebraic Sato-Tate group considered in sections $\S 4-\S 6$, in particular in all cases where the MumfordTate group is determined by endomorphisms.

\section{The algebraic Sato-Tate group and conjecture}

We begin with the definition of the algebraic Sato-Tate group and the formulation of an associated conjecture refining the standard Mumford-Tate conjecture.

Definition 2.1. Let $A / F$ be an abelian variety over a number field $F$. Choose an embedding $F \subset \mathbb{C}$, then put $V:=V(A):=H_{1}(A(\mathbb{C}), \mathbb{Q})$ and $V^{*}:=\operatorname{Hom}_{\mathbb{Q}}(V, \mathbb{Q})$. Put $D:=D(A):=\operatorname{End}(A)^{0}:=\operatorname{End}_{\bar{F}}(A) \otimes \mathbb{Q}$.

We obtain a rational Hodge structure of weight 1 on $V^{*}$ from the Hodge decomposition

$$
V_{\mathbb{C}}^{*}:=V^{*} \otimes_{\mathbb{Q}} \mathbb{C}=H^{1,0} \oplus H^{0,1},
$$


where $H^{p, q}=H^{p}\left(A ; \Omega_{A / \mathbb{C}}^{q}\right)$ and $\overline{H^{p, q}}=H^{q, p}$. Observe that $H^{p, q}$ are invariant subspaces with respect to the action of $D$ on $V^{*} \otimes_{\mathbb{Q}} \mathbb{C}$, so the $H^{p, q}$ are right $D$ modules. Put $H^{-p,-q}:=\operatorname{Hom}_{\mathbb{C}}\left(H^{p, q}, \mathbb{C}\right)$. The left action of $D$ on $H^{-p,-q}$ is defined via the right action of $D$ on $H^{p, q}$. Let

$$
\psi=\psi_{V}: V \times V \rightarrow \mathbb{Q}
$$

be the $\mathbb{Q}$-bilinear, nondegenerate, alternating form coming from the Riemann form of $A$.

Definition 2.2. Put $T_{l}:=T_{l}(A)$ and $V_{l}:=V_{l}(A):=T_{l}(A) \otimes_{\mathbb{Z}_{l}} \mathbb{Q}_{l}$. It is well known [Md, $\S 24$, p. 237] (cf. [Mi, §16, p. 133]) that $V_{l} \cong V \otimes_{\mathbb{Q}} \mathbb{Q}_{l}$. The Galois group $G_{F}$ acts naturally on $V_{l}$ and because of the Weil pairing $\psi_{l}: V_{l} \times V_{l} \rightarrow \mathbb{Q}_{l}$ we have the $l$-adic representation

$$
\rho_{l}: G_{F} \rightarrow G S p\left(V_{l}\right)
$$

induced by the natural representation $\rho_{l}: G_{F} \rightarrow G S p\left(T_{l}\right)$.

Let $K / F$ be a finite extension. Let $G_{l, K}^{a l g} \subseteq G S p_{\left(V_{l}, \psi_{l}\right)}$ be the Zariski closure of $\rho_{l}\left(G_{K}\right)$ in $G S p_{\left(V_{l}, \psi_{l}\right)}$. Define

$$
\rho_{l}\left(G_{K}\right)_{1}:=\rho_{l}\left(G_{K}\right) \cap S p_{\left(V_{l}, \psi_{l}\right)} .
$$

Let $G_{l, K, 1}^{a l g} \subseteq S p_{\left(V_{l}, \psi_{l}\right)}$ be the Zariski closure of $\rho_{l}\left(G_{K}\right)_{1}$ in $S p_{\left(V_{l}, \psi_{l}\right)}$. The algebraic group $G_{l, K}^{\text {alg }}$ is reductive by [F, Theorem 3]. If $K=F$, we will put $G_{l}^{a l g}:=G_{l, F}^{a l g}$ and $G_{l, 1}^{a l g}:=G_{l, F, 1}^{a l g}$.

On the basis of the work of Mumford, Serre, and Tate, it has been conjectured for about 50 years (see Conjecture 4.7) that there is a connected reductive group scheme $G \subseteq G S p_{(V, \psi)}$ such that for all $l \gg 0$,

$$
G_{\mathbb{Q}_{l}}=\left(G_{l, K}^{a l g}\right)^{\circ},
$$

where $\left(G_{l, K}^{a l g}\right)^{\circ}$ denotes the connected component of the identity. This implies that $G$ does not depend on $K$ (see 93 ). We propose the following refinement of this conjecture which does detect the field $K$.

Conjecture 2.3. (Algebraic Sato-Tate conjecture) There is an algebraic group $A S T_{K}(A) \subseteq S p_{(V, \psi)}$ over $\mathbb{Q}$ such that $A S T_{K}(A)^{\circ}$ is reductive and for each $l$,

$$
A S T_{K}(A)_{\mathbb{Q}_{l}}=G_{l, K, 1}^{a l g} .
$$

Definition 2.4. The algebraic Sato-Tate group plays a key role in Serre's definition of the putative Sato-Tate group $S T_{K}(A)$. If Conjecture 2.3 holds, then base extension of $A S T_{K}(A)_{\mathbb{Q}_{l}}$ along an embedding $\mathbb{Q}_{l} \subset \mathbb{C}$ gives a group $A S T_{K}(A)_{\mathbb{C}}$ that does not depend on $l$ or the embedding. Taking a maximal compact subgroup of $A S T_{K}(A)(\mathbb{C})$, we obtain the Sato-Tate group $S T_{K}(A) \subseteq U S p\left(V_{\mathbb{C}}\right)$.

Remark 2.5. Besides Conjecture 2.3 and the usual Sato-Tate conjecture, we expect $A S T_{K}(A)$ to have the following properties:

$$
\begin{gathered}
A S T_{K}(A) \subseteq D L_{K}(A) \\
A S T_{K}(A)^{\circ}=H(A) \\
\pi_{0}\left(A S T_{K}(A)\right)=\pi_{0}\left(S T_{K}(A)\right) .
\end{gathered}
$$


Here $H(A)$ is the Hodge group and $D L_{K}(A)$ is the twisted decomposable Lefschetz group, to be defined in $\S 5$. In $\S 5$, we exhibit a wide class of abelian varieties such that Conjecture 2.3 and conditions (2.4)-(2.6) hold (see Theorem 6.1).

In $\S 9$, we define a candidate group $A S T_{K}(A)$ based on computations in $\S 7$ and $\S 8$ using the Tannakian formalism. Assuming that the Mumford-Tate group is the connected component of the motivic Mumford-Tate group (a hypothesis put forward by Serre in [Se4, sec. 3.4]), we prove (see Theorem [9.8) that the group $A S T_{K}(A)$ satisfies conditions (2.4)-(2.6).

\section{Some remarks on Zariski closure. A theorem of Serre.}

Before proceeding further, we collect some observations about the groups we have just defined.

Remark 3.1. We have the following commutative diagram with exact columns:

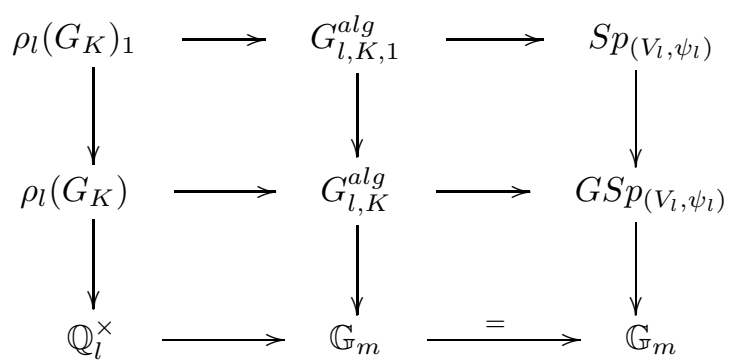

By the theorem of Bogomolov [Bo] on homotheties, $\rho_{l}\left(G_{K}\right)$ is an open subgroup of $G_{l, K}^{a l g}$. Since $S p_{\left(V_{l}, \psi_{l}\right)}$ is closed in $G S p_{\left(V_{l}, \psi_{l}\right)}, \rho_{l}\left(G_{K}\right)_{1}=\rho_{l}\left(G_{K}\right) \cap S p_{\left(V_{l}, \psi_{l}\right)}$ is an open subgroup of $G_{l, K}^{a l g} \cap S p_{\left(V_{l}, \psi_{l}\right)}$. It follows that

$$
G_{l, K, 1}^{a l g}=G_{l, K}^{a l g} \cap S p_{\left(V_{l}, \psi_{l}\right)}
$$

is closed in $G_{l, K}^{a l g}$, and that there is an exact sequence

$$
1 \rightarrow G_{l, K, 1}^{a l g} \rightarrow G_{l, K}^{a l g} \rightarrow \mathbb{G}_{m} \rightarrow 1 .
$$

Since $G_{l, K, 1}^{a l g}$ is the kernel of a homomorphism from a reductive group to a torus, it is also reductive.

Remark 3.2. Let $F \subseteq K \subseteq L \subset \bar{F}$ be a chain of extensions such that $K / F$ and $L / K$ are finite. Let $L^{\prime} \subset \bar{F}$ be the Galois closure of $L$ over $K$. It is clear that $G_{l, L^{\prime}}^{a l g} \subseteq G_{l, L}^{a l g} \subseteq G_{l, K}^{a l g}$ and $G_{l, L^{\prime}, 1}^{\text {alg }} \subseteq G_{l, L, 1}^{a l g} \subseteq G_{l, K, 1}^{a l g}$. On the other hand, there is a surjective homomorphism $\rho_{l}\left(G_{K}\right) / \rho_{l}\left(G_{L^{\prime}}\right) \rightarrow G_{l, K}^{a l g} / G_{l, L^{\prime}}^{a l g}$, so $G_{l, L^{\prime}}^{a l g}$ is a subgroup of $G_{l, K}^{a l g}$ of finite index, as then is $G_{l, L}^{a l g}$. In particular, $\left(G_{l, L}^{a l g}\right)^{\circ}=\left(G_{l, K}^{a l g}\right)^{\circ}$. By a similar argument, $\left(G_{l, L, 1}^{a l g}\right)^{\circ}=\left(G_{l, K, 1}^{a l g}\right)^{\circ}$.

Proposition 3.3. There is a finite Galois extension $L_{0} / K$ such that $G_{l, L_{0}}^{a l g}=$ $\left(G_{l, K}^{a l g}\right)^{\circ}$ and $G_{l, L_{0}, 1}^{a l g}=\left(G_{l, K, 1}^{a l g}\right)^{\circ}$.

Proof. Let $Z$ be the set of $\mathbb{Q}_{l}$-points of the closed subscheme $\left(G_{l, K}^{a l g}-\left(G_{l, K}^{a l g}\right)^{\circ}\right) \cup$ $\left(G_{l, K, 1}^{a l g}-\left(G_{l, K, 1}^{a l g}\right)^{\circ}\right)$ of $G S p_{\left(V_{l}, \psi_{l}\right)}$. Since $\rho_{l}$ is continuous, we can find a finite Galois 
extension $L_{0} / K$ such that $\rho_{l}\left(G_{L_{0}}\right) \cap Z=\emptyset$. Such an extension satisfies $G_{l, L_{0}}^{a l g} \subseteq$ $\left(G_{l, K}^{a l g}\right)^{\circ}$ and $G_{l, L_{0}, 1}^{a l g} \subseteq\left(G_{l, K, 1}^{a l g}\right)^{\circ}$. Since we already have the reverse inclusions, we obtain the desired equalities.

The following theorem is a special case of a result of Serre [Se5, §8.3.4].

Theorem 3.4. The following map is an isomorphism:

$$
i_{C C}: G_{l, K, 1}^{a l g} /\left(G_{l, K, 1}^{a l g}\right)^{\circ} \stackrel{\cong}{\longrightarrow} G_{l, K}^{a l g} /\left(G_{l, K}^{a l g}\right)^{\circ} .
$$

Proof. Choose $L_{0}$ as in Proposition 3.3 Then the following commutative diagram has exact rows:

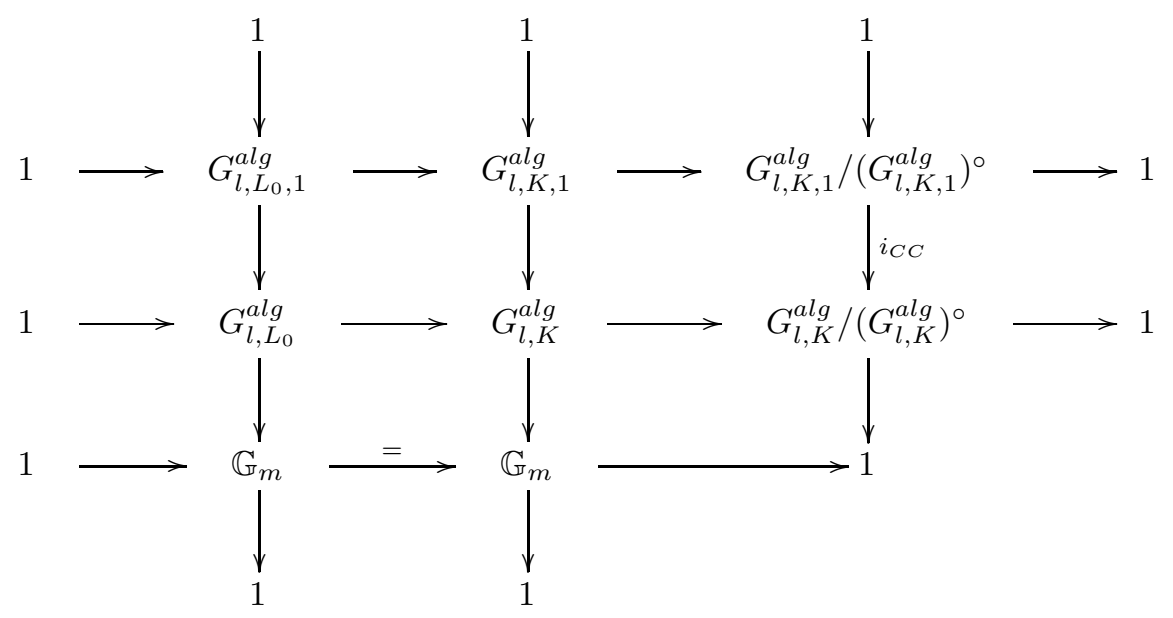

The first two columns are also exact by (3.2), so a diagram chase (as in the snake lemma) shows that the third column is also exact.

Remark 3.5. Observe that the natural action by left translation

$$
G_{K} \times G_{l, K}^{a l g} /\left(G_{l, K}^{a l g}\right)^{\circ} \rightarrow G_{l, K}^{a l g} /\left(G_{l, K}^{a l g}\right)^{\circ}
$$

is continuous. By Theorem 3.4, we also have a continuous action

$$
G_{K} \times G_{l, K, 1}^{a l g} /\left(G_{l, K, 1}^{a l g}\right)^{\circ} \rightarrow G_{l, K, 1}^{a l g} /\left(G_{l, K, 1}^{\text {alg }}\right)^{\circ} .
$$

Choose a suitable field embedding $\mathbb{Q}_{l} \rightarrow \mathbb{C}$ and put $G_{l, K, 1 \mathbb{C}}^{a l g}:=G_{l, K, 1}^{a l g} \otimes_{\mathbb{Q}_{l}} \mathbb{C}$. By Theorem 3.4 and [?, Lemma 2.8], we have the following.

Proposition 3.6. Let $K / F$ be any finite extension such that $F \subseteq K \subset \bar{F}$. Then there are natural isomorphisms

$$
G_{l, K, 1}^{a l g} /\left(G_{l, K, 1}^{a l g}\right)^{\circ} \cong G_{l, K, 1_{\mathbb{C}}}^{a l g} /\left(G_{l, K, 1 \mathbb{C}}^{a l g}\right)^{\circ} \cong S T_{A} / S T_{A}^{\circ} .
$$

If the algebraic Sato-Tate conjecture (Conjecture 2.3) holds, then all of the groups in (3.5) are isomorphic to $A S T_{K}(A) / A S T_{K}(A)^{\circ}$.

Remark 3.7. Consider the continuous homomorphism

$$
\epsilon_{l, K}: G_{K} \rightarrow G_{l, K}^{a l g}\left(\mathbb{Q}_{l}\right) .
$$

Serre [Se3], [Se2] proved that $\epsilon_{l, K}^{-1}\left(\left(G_{l, K}^{a l g}\right)^{\circ}\left(\mathbb{Q}_{l}\right)\right)$ is independent of $l$. Since $\left(G_{l, K}^{a l g}\right)^{\circ}$ is open in $G_{l, K}^{\text {alg }}$, this preimage equals $G_{L_{0}}=G\left(\bar{K} / L_{0}\right)$ for some finite Galois extension 
$L_{0} / K$. This theorem of Serre can also be seen from Propositions 3.3 and 3.6 and Theorem 3.4. In fact, $L_{0} / K$ is the minimal extension such that $G_{l, L_{0}}^{a l g}$ and $G_{l, L_{0}, 1}^{a l g}$ are connected for all $l$. Let $H_{l, K, 1}:=\rho_{l}^{-1}\left(\rho_{l}\left(G_{K}\right)_{1}\right)$. Put $K_{1}:=\bar{K}^{H_{l, K, 1}}$. Observe that

$$
\begin{gathered}
\epsilon_{l, K}^{-1}\left(\left(G_{l, K, 1}^{a l g}\right)^{\circ}\left(\mathbb{Q}_{l}\right)\right)=\epsilon_{l, K}^{-1}\left(G_{l, L_{0}, 1}^{a l g}\left(\mathbb{Q}_{l}\right)\right)=\epsilon_{l, K}^{-1}\left(\left(G_{l, L_{0}}^{a l g} \cap S p_{\left(V_{l}, \psi_{l}\right)}\right)\left(\mathbb{Q}_{l}\right)\right)= \\
=\epsilon_{l, K}^{-1}\left(G_{l, L_{0}}^{a l g}\left(\mathbb{Q}_{l}\right)\right) \cap \epsilon_{l, K}^{-1}\left(\operatorname{Sp}_{\left(V_{l}, \psi_{l}\right)}\left(\mathbb{Q}_{l}\right)\right)=G_{L_{0}} \cap G_{K_{1}}=G_{L K_{1}} .
\end{gathered}
$$

\section{Mumford-Tate group and Mumford-Tate conjecture}

We next recall some standard definitions and results concerning the MumfordTate group. We continue to assume that $A$ is an abelian variety over a number field $F$ equipped with a fixed embedding into $\mathbb{C}$.

Definition 4.1. For $V=H_{1}(A, \mathbb{Q})$, define the cocharacter

$$
\mu_{\infty, A}: \mathbb{G}_{m}(\mathbb{C}) \rightarrow G L\left(V_{\mathbb{C}}\right)
$$

such that for any $z \in \mathbb{C}^{\times}$, the automorphism $\mu_{\infty, A}(z)$ acts as multiplication by $z$ on $H^{-1,0}$ and as the identity on $H^{0,-1}$. Observe that by definition we have $\mu_{\infty, A}\left(\mathbb{C}^{\times}\right) \subset G S p_{(V, \psi)}(\mathbb{C})$.

Definition 4.2. (Mumford-Tate and Hodge groups)

(1) The Mumford-Tate group of $A / F$ is the smallest algebraic subgroup $M T(A) \subseteq$ $G L_{V}$ over $\mathbb{Q}$ such that $M T(A)(\mathbb{C})$ contains $\mu_{\infty}(\mathbb{C})$.

(2) The decomposable Hodge group is $D H(A):=M T(A) \cap S L_{V}$.

(3) The Hodge group $H(A):=D H(A)^{\circ}$ is the connected component of the identity in $D H(A)$.

By definition, if $K / F$ is a field extension such that $K \subset \bar{F}$, then $M T(A)$ and $H(A)$ for $A / F$ and for $A / K$ are the same. That is, we do not need to include $K$ in the notation.

Remark 4.3. Note that $M T(A)$ is a reductive subgroup of $G S p_{(V, \psi)}$ D2, Prop. 3.6], $H(A) \subseteq S p_{(V, \psi)}$, and $M T(A) \subseteq C_{D}\left(G S p_{(V, \psi)}\right)$. Hence

$$
H(A) \subseteq C_{D}\left(S p_{(V, \psi)}\right) .
$$

Definition 4.4. The algebraic group $L(A)=C_{D}^{\circ}\left(S p_{(V, \psi)}\right)$ is called the Lefschetz groun 1 of $A$. By (4.1) and the connectedness of $H(A)$, we have $H(A) \subseteq L(A)$.

Definition 4.5. For a field extension $L / \mathbb{Q}$ put

$$
\begin{gathered}
M T(A)_{L}:=M T(A) \otimes_{\mathbb{Q}} L, \quad D H(A)_{L}:=D H(A) \otimes_{\mathbb{Q}} L, \\
H(A)_{L}:=H(A) \otimes_{\mathbb{Q}} L, \quad L(A)_{L}:=L(A) \otimes_{\mathbb{Q}} L .
\end{gathered}
$$

Theorem 4.6. (Deligne D2, I, Prop. 6.2]) For any prime number $l$,

$$
\left(G_{l}^{a l g}\right)^{\circ} \subseteq M T(A)_{\mathbb{Q}_{l}} .
$$

Conjecture 4.7. (Mumford-Tate) For any prime number $l$,

$$
\left(G_{l}^{a l g}\right)^{\circ}=M T(A)_{\mathbb{Q}_{l}} .
$$

\footnotetext{
${ }^{1}$ This terminology is due to Murty $\mathrm{My}$.
} 
Remark 4.8. Observe that (4.2) is equivalent to the inclusion

$$
\left(G_{l, K, 1}^{a l g}\right)^{\circ} \subseteq H(A)_{\mathbb{Q}_{l}},
$$

while the Mumford-Tate conjecture is equivalent to the equality

$$
\left(G_{l, K, 1}^{a l g}\right)^{\circ}=H(A)_{\mathbb{Q}_{l}} .
$$

This follows immediately from the following commutative diagram in which every column is exact and every horizontal arrow is a containment of corresponding group schemes.

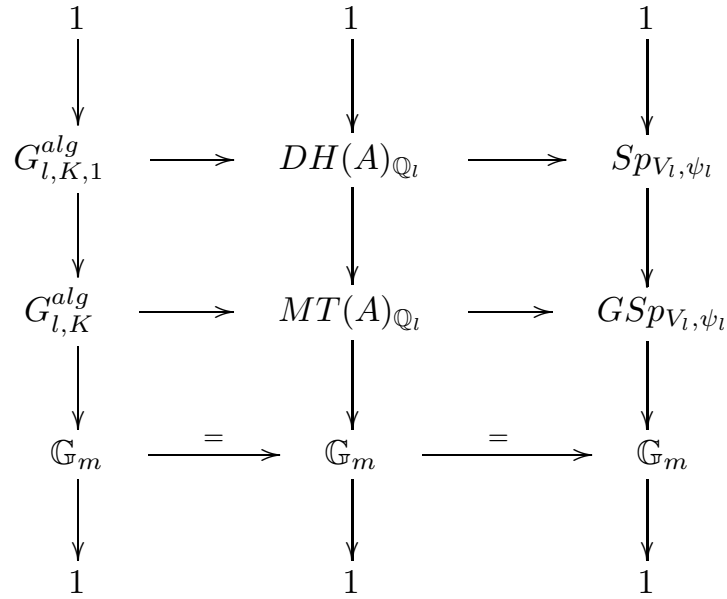

It is known that the Mumford-Tate and Hodge groups do not behave well in general with respect to products of abelian varieties [G. p. 316]. However one can prove the following theorem.

Theorem 4.9. The Mumford-Tate group of abelian varieties have the following properties.

1. An isogeny $\phi: A_{1} \rightarrow A_{2}$ of abelian varieties induces isomorphisms $M T\left(A_{1}\right) \cong$ $M T\left(A_{2}\right)$ and $H\left(A_{1}\right) \cong H\left(A_{2}\right)$.

2. If $A$ is an abelian variety and $A^{s}:=\prod_{i=1}^{s} A$, then $M T\left(A^{s}\right) \cong M T(A)$ and $H\left(A^{s}\right) \cong H(A)$.

Proof. 1. This follows because an isogeny induces an isomorphism of associated polarised rational Hodge structures.

2. Observe that there is a natural isomorphism $H^{1}\left(A^{s}(\mathbb{C}), \mathbb{Q}\right) \cong \oplus_{i=1}^{s} H^{1}(A(\mathbb{C}), \mathbb{Q})$ or simply $V\left(A^{s}\right) \cong V(A)^{s}$. So in this case, the Hodge structure on $H^{1}\left(A^{s}(\mathbb{C}), \mathbb{Q}\right)$ is isomorphic to the direct sum of the Hodge structures on $H^{1}(A(\mathbb{C}), \mathbb{Q})$. Via this identification, we have $\mu_{\infty, A^{s}}=\Delta\left(\mu_{\infty, A}\right)$ where $\Delta: G S p\left(V_{\mathbb{C}}\right) \rightarrow G S p\left(V_{\mathbb{C}}\right)^{s} \subseteq G L\left(V_{\mathbb{C}}^{s}\right)$ is the diagonal homomorphism. So we can consider $M T\left(A^{s}\right)$ as a subgroup of $M T(A)^{s}:=\prod_{i=1}^{s} M T(A)$. Consider the diagonal homomorphism $\Delta: M T(A) \rightarrow$ $M T(A)^{s}$. Since $\mu_{\infty, A^{s}}\left(\mathbb{G}_{m}(\mathbb{C})\right)=\Delta\left(\mu_{\infty, A}\right)\left(\mathbb{G}_{m}(\mathbb{C})\right) \subseteq \Delta(M T(A))(\mathbb{C})$, we have $M T\left(A^{s}\right) \subseteq \Delta(M T(A)) \cong M T(A)$. Since $M T\left(A^{s}\right)(\mathbb{C})$ contains $\Delta\left(\mu_{\infty, A}\left(\mathbb{G}_{m}(\mathbb{C})\right)\right)$ then the image of $M T\left(A^{s}\right)$ via projection $\pi: M T(A)^{s} \rightarrow M T(A)$ on every factor contains the image of $\mu_{\infty, A}\left(\mathbb{G}_{m}(\mathbb{C})\right)$. By the definition of $M T(A)$, this means that the projection $\pi$ must surject onto $M T(A)$. On the other hand, since $M T\left(A^{s}\right) \subseteq \Delta(M T(A))$, it is clear that for $\pi$ to be onto, we must have $M T\left(A^{s}\right)=$ 
$\Delta(M T(A))$. To get the isomorphism of Hodge groups, we note that the isomorphism $V\left(A^{s}\right) \cong V(A)^{s}$ gives an isomorphism $S L_{V\left(A^{s}\right)} \cong S L_{V(A)^{s}}$. We thus have the following isomorphisms of algebraic groups over $\mathbb{Q}$ :

$$
\begin{aligned}
& M T\left(A^{s}\right) \cap S L_{V\left(A^{s}\right)} \cong \Delta(M T(A)) \cap S L_{V(A)^{s}} \cong \\
& \cong \Delta(M T(A)) \cap\left(S L_{V(A)}\right)^{s} \cong M T(A) \cap S L_{V(A)} .
\end{aligned}
$$

Taking connected components, we get the desired isomorphism of Hodge groups.

One can make a corresponding calculation also on the Galois side.

Theorem 4.10. We have the following results.

1. An isogeny $\phi: A_{1} \rightarrow A_{2}$ of abelian varieties over a number field $K$ induces isomorphisms $G_{l, K}^{a l g}\left(A_{1}\right) \cong G_{l, K}^{\text {alg }}\left(A_{2}\right)$ and $G_{l, K, 1}^{\text {alg }}\left(A_{1}\right) \cong G_{l, K, 1}^{\text {alg }}\left(A_{2}\right)$.

2. If $A$ is an abelian variety over a number field $K$, then for any positive integer $s, G_{l, K}^{a l g}\left(A^{s}\right) \cong G_{l, K}^{\text {alg }}(A)$ and $G_{l, K, 1}^{a l g}\left(A^{s}\right)=G_{l, K, 1}^{a l g}(A)$.

Proof. 1. Observe that an isogeny $\phi$ induces an isomorphism of Galois modules $V_{l}\left(A_{1}\right) \cong V_{l}\left(A_{2}\right)$.

2. The natural isomorphism $V_{l}\left(A^{s}\right) \cong V_{l}(A)^{s}$ of $G_{K}$-modules shows that $\rho_{l, A^{s}} \cong$ $\Delta \rho_{l, A}$, where $\Delta \rho_{l, A}: G_{K} \rightarrow G S p\left(V_{l}(A)\right)^{s}$ is the natural diagonal representation $\Delta \rho_{l, A}=\operatorname{diag}\left(\rho_{l, A}, \ldots, \rho_{l, A}\right)$.

Corollary 4.11. Let $A / K$ be an abelian variety such that the Mumford-Tate conjecture holds for A. Then the Mumford-Tate conjecture holds for $A^{s}$ for any positive integer $s$.

Proof. It follows from Theorems 4.9 and 4.10

Remark 4.12. Observe that if the Mumford-Tate conjecture holds for $A$ and $K$ is a finite extension of $F$ for which $G_{l, K}^{a l g}$ is connected, then

$$
G_{l, K, 1}^{a l g}\left(A^{s}\right)=H\left(A^{s}\right)_{\mathbb{Q}_{l}} .
$$

for any $s \geq 1$. Hence the algebraic Sato-Tate conjecture holds for $A^{s}$ for any $s \geq 1$ with

$$
A S T_{K}\left(A^{s}\right)=H\left(A^{s}\right)
$$

\section{Twisted Lefschetz groups and the algebraic Sato-Tate Conjecture}

We next use the Lefschetz group to define an upper bound on the algebraic SatoTate group. In those cases where the Mumford-Tate group is determined entirely by endomorphisms, this allows us to deduce the algebraic Sato-Tate conjecture from the Mumford-Tate conjecture. (An analogous construction for modular forms is the twisting field appearing in the work of Momose $[\mathrm{Mo}$ and Ribet $[\mathrm{R}$.) We continue to take $A$ to be an abelian variety over a number field $F$ embedded in $\mathbb{C}$ and $K$ to be a finite extension of $F$.

Definition 5.1. Note that we have a continuous representation

$$
\rho_{e}: G_{K} \rightarrow \operatorname{Aut}_{\mathbb{Q}}(D)
$$


Put $G_{L_{e}}:=\operatorname{Ker} \rho_{e}$, so that $L_{e} / K$ is a finite Galois extension. For $\tau \in G\left(L_{e} / K\right)$, define

$$
D L_{K}^{\tau}(A):=\left\{g \in S p_{V}: g \beta g^{-1}=\rho_{e}(\tau)(\beta) \forall \beta \in D\right\} .
$$

It is enough to impose the condition for $\beta$ running over a $\mathbb{Q}$-basis of $D$; therefore, $D L_{K}^{\tau}(A)$ is a closed subscheme of $S p_{V}$ for each $\tau$.

Definition 5.2. Define the twisted decomposable algebraic Lefschetz group of $A$ over $K$ to be the closed algebraic subgroup of $S p_{V, \psi}$ given by

$$
D L_{K}(A)=\bigsqcup_{\tau \in G\left(L_{e} / K\right)} D L_{K}^{\tau}(A) .
$$

For any subextension $L / K$ of $\bar{F} / K$ we have $D L_{L}(A) \subseteq D L_{K}(A)$ and $D L_{L}^{i d}(A)=$ $D L_{K}^{i d}(A)$. Hence the classical Lefschetz group of $A$ can be written as:

$$
L(A)=D L_{K}^{i d}(A)^{\circ}=D L_{K}(A)^{\circ} .
$$

and $L(A)=D L_{L}^{i d}(A)^{\circ}=D L_{L}(A)^{\circ}$, for any subextension $L / K$ of $\bar{F} / K$. In particular $D L_{L_{e}}^{i d}(A)=D L_{L_{e}}(A)=D L_{\bar{F}}(A)=D L_{\bar{F}}^{i d}(A)$ and $L(A)=D L_{L_{e}}(A)^{\circ}=$ $D L_{\bar{F}}(A)^{\circ}$.

Theorem 5.3. The twisted decomposable Lefschetz groups of abelian varieties have the following properties.

1. An isogeny $\phi: A_{1} \rightarrow A_{2}$ of abelian varieties over $K$ induces an isomorphism $D L_{K}^{\tau}\left(A_{1}\right) \cong D L_{K}^{\tau}\left(A_{2}\right)$ for every $\tau \in G\left(L_{e} / K\right)$, and consequently an isomorphism $D L_{K}\left(A_{1}\right) \cong D L_{K}\left(A_{2}\right)$.

2. If $A / K$ is an abelian variety, then $D L_{K}^{\tau}\left(A^{s}\right) \cong D L_{K}^{\tau}(A)$ for every $\tau \in$ $G\left(L_{e} / K\right)$.

3. If $A=\prod_{i=1}^{t} A_{i}$ with $A_{i} / K$ simple for all $i$ and $\operatorname{Hom}_{\bar{F}}\left(A_{i}, A_{j}\right)=0$ for all $i \neq j$, then $D L_{K}^{\tau}(A) \cong \prod_{i=1}^{t} D L_{K}^{\tau}\left(A_{i}\right)$ for every $\tau \in G\left(L_{e} / K\right)$.

4. If $A=\prod_{i=1}^{t} A_{i}^{s_{i}}$ with $A_{i} / K$ for all $i$ and $\operatorname{Hom}_{\bar{F}}\left(A_{i}, A_{j}\right)=0$ for all $i \neq j$, then $D L_{K}^{\tau}(A) \cong \prod_{i=1}^{t} D L_{K}^{\tau}\left(A_{i}\right)$ for every $\tau \in G\left(L_{e} / K\right)$.

5. If $A=\prod_{i=1}^{t} A_{i}^{s_{i}}$ with all $A_{i} / K$ simple, pairwise nonisogenous then $D L_{K}^{\tau}(A) \cong$ $\prod_{i=1}^{t} D L_{K}^{\tau}\left(A_{i}\right)$ for every $\tau \in G\left(L_{e} / K\right)$.

Proof. 1. An isogeny induces the isomorphism $\left(V\left(A_{1}\right), \psi_{V\left(A_{1}\right)}\right) \cong\left(V\left(A_{2}\right), \psi_{V\left(A_{2}\right)}\right)$ of $\mathbb{Q}$-bilinear spaces. It also induces an isomorphism $D\left(A_{1}\right)=\operatorname{End}_{\bar{K}}\left(A_{1}\right) \otimes_{\mathbb{Z}} \mathbb{Q} \cong$ $D\left(A_{2}\right)=\operatorname{End}_{\bar{K}}\left(A_{2}\right) \otimes \mathbb{Q}$ of $\mathbb{Q}\left[G_{K}\right]$-modules, and so the first claim follows.

2. There is a natural isomorphism:

$$
\left(V\left(A^{s}\right), \psi_{V\left(A^{s}\right)}\right) \cong\left(V(A), \psi_{V(A)}\right)^{s}:=\bigoplus_{i=1}^{s}\left(V(A), \psi_{V(A)}\right)
$$

and an isomorphism $D\left(A^{s}\right) \cong M_{s}(D(A))$ of $\mathbb{Q}$-algebras. Let $\Delta$ be the homomorphism that maps $S p_{\left(V(A), \psi_{V(A)}\right)} \operatorname{onto} \operatorname{diag}\left(S p_{\left(V(A), \psi_{V(A)}\right)}, \ldots, S p_{\left(V(A), \psi_{V(A)}\right)}\right) \subseteq$

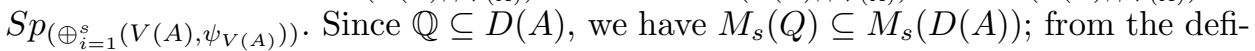
nition of the twisted decomposable Lefschetz group, we get $D L_{K}^{\tau}\left(A^{s}\right) \cong \Delta\left(D L_{K}^{\tau}(A)\right) \cong$ $D L_{K}^{\tau}(A)$.

3. The proof is similar to the proof of 2 . under the observation that $D(A) \cong$ $\prod_{i=1}^{t} D\left(A_{i}\right)$ and $\left(V(A), \psi_{V(A)}\right) \cong \bigoplus_{i=1}^{t}\left(V\left(A_{i}\right), \psi_{V\left(A_{i}\right)}\right)$.

4. This follows immediately from 2 . and 3 .

5. Immediate corollary from 4. 
Remark 5.4. Theorem 5.3 remains true if we replace $D L_{K}^{\tau}(B)$ with $D L_{K}^{\tau}(B)^{\circ}$ for all abelian varieties $B$ that appear in the theorem. Since $L(B)=D L_{K}^{i d}(B)^{\circ}$, then the Lefschetz group satisfies properties $1-5$ of Theorem 5.3. The property 5 . of this theorem in the case of the Lefschetz group was obtained previously by K. Murty My, Lemma 2.1], cf. [G, Lemma B.70].

Remark 5.5. Observe that we have

$$
D L_{K}(A):=\left\{g \in S p_{(V, \psi)}: \exists_{\tau \in G_{K}} \forall \beta \in D \quad g \beta g^{-1}=\rho_{e}(\tau)(\beta)\right\}
$$

Changing quantifiers we get another group scheme

$$
\widetilde{D L_{K}(A)}:=\left\{g \in S p_{(V, \psi)}: \forall_{\beta \in D} \exists_{\tau \in G_{K}} \quad g \beta g^{-1}=\rho_{e}(\tau)(\beta)\right\}
$$

Observe that $D L_{K}(A) \subseteq \widetilde{D L_{K}(A)}$.

We now use the twisted Lefschetz group to obtain an upper bound on the algebraic Sato-Tate group.

Remark 5.6. Observe that (4.1) implies that

$$
D H(A) \subseteq D L_{K}^{i d}(A) \subseteq D L_{K}(A) .
$$

On the other hand, for any $P \in A(\bar{F})$, any $\beta \in \operatorname{End}_{\bar{F}}(A)$, and any $\sigma \in G_{K}$, we have $\sigma \beta \sigma^{-1}(P)=\sigma(\beta)(P)$. Hence $\rho_{l}\left(G_{K}\right)_{1} \subseteq D L_{K}(A)\left(\mathbb{Q}_{l}\right)$. In particular,

$$
G_{l, K, 1}^{a l g} \subseteq D L_{K}(A)_{\mathbb{Q}_{l}} .
$$

We will be particularly interested in cases where $G_{l, K, 1}^{a l g}=D L_{K}(A)_{\mathbb{Q}_{l}}$. We next check that this condition does not depend on the field $K$.

Remark 5.7. Let $\tilde{\tau} \in G_{K}$ be a lift of $\tau \in G\left(L_{e} / K\right)$. The coset $\tilde{\tau} G_{L_{e}}$ does not depend on the lift. The Zariski closure of $\rho_{l}\left(\tilde{\tau} G_{L_{e}}\right)=\rho_{l}(\tilde{\tau}) \rho_{l}\left(G_{L_{e}}\right)$ in $S p_{V_{l}}$ is

$$
\rho_{l}(\tilde{\tau}) G_{l, L_{e}, 1}^{a l g} \subseteq D L_{K}^{\tau}(A)_{\mathbb{Q}_{l}} .
$$

We observe that

$$
G_{l, K, 1}^{a l g}=\bigsqcup_{\tau \in G\left(L_{e} / K\right)} \rho_{l}(\tilde{\tau}) G_{l, L_{e}, 1}^{a l g} .
$$

Since $D L_{K}^{i d}(A)=D L_{L_{e}}(A)=D L_{\bar{F}}(A)$, we get

$$
G_{l, L_{e}, 1}^{a l g} \subseteq D L_{L_{e}}(A)_{\mathbb{Q}_{l}} .
$$

Hence the following two equalities are equivalent:

$$
\begin{aligned}
G_{l, L_{e}, 1}^{a l g} & =D L_{L_{e}}(A)_{\mathbb{Q}_{l}}, \\
G_{l, K, 1}^{a l g} & =D L_{K}(A)_{\mathbb{Q}_{l}} .
\end{aligned}
$$

\section{Some cases of the algebraic Sato-Tate conjecture}

In general, the containment $H(A) \subseteq L(A)$ can be strict, which makes the Mumford-Tate conjecture a somewhat subtle problem; for instance, Mumford exhibited examples of simple abelian fourfolds for which $H(A) \neq L(A)$. Fortunately, when $A$ has a large endomorphism algebra as compared to its dimension, such pathologies do not occur: one has $H(A)=L(A)$ and one can often show that the Mumford-Tate conjecture holds. When this happens, we will say that the Mumford-Tate conjecture for $A$ is explained by endomorphisms. 
The following theorem asserts that in cases where the Mumford-Tate conjecture is explained by endomorphisms and the twisted decomposable Lefschetz group over $\bar{F}$ is connected, the algebraic Sato-Tate conjecture is in a sense also explained by endomorphisms.

Theorem 6.1. Assume that the following conditions hold.

1. We have $H(A)=L(A)$.

2. We have $\left(G_{l, K}^{\text {alg }}\right)^{\circ}=M T(A)_{\mathbb{Q}_{l}}$.

3. The group $D L_{\bar{F}}(A)$ is connected.

Then (5.12) holds for every l. Consequently, the algebraic Sato-Tate conjecture (Conjecture 2.3) holds for $A / K$ with

$$
A S T_{K}(A)=D L_{K}(A) .
$$

Proof. It is enough to prove (5.11). By our assumptions and Remark 4.8 we get $\left(G_{l, L_{e}, 1}^{a l g}\right)^{\circ}=H(A)_{\mathbb{Q}_{l}}=L(A)_{\mathbb{Q}_{l}}=D L_{L_{e}}(A)_{\mathbb{Q}_{l}}$. Hence $D L_{L_{e}}(A)_{\mathbb{Q}_{l}}$ is also connected for every $l$ and by (5.10) we obtain $\left(G_{l, L_{e}, 1}^{a l g}\right)^{\circ}=G_{l, L_{e}, 1}^{a l g}$ for every $l$.

Remark 6.2. Under assumptions of Theorem 6.1 the results of Theorems 4.9, 4.10 and 5.3 show that the algebraic Sato-Tate conjecture holds for $A^{s}$ for all $s \geq 1$ with $A S T_{K}\left(A^{s}\right)=D L_{K}\left(A^{s}\right) \cong D L_{K}(A)=A S T_{K}(A)$.

Conversely, if the algebraic Sato-Tate conjecture for $A$ is explained by endomorphisms, so is the Mumford-Tate conjecture.

Theorem 6.3. Assume that (5.12) and (6.1) hold for every $l$ (so in particular, the algebraic Sato-Tate conjecture holds). We then have the following.

1. We have $H(A)=L(A)$.

2. We have $\left(G_{l, K}^{\text {alg }}\right)^{\circ}=M T(A)_{\mathbb{Q}_{l}}$.

Proof. By Theorem 4.6 and Remark 4.8 (see (4.4)), we have

$$
\left(G_{l, K, 1}^{a l g}\right)^{\circ} \subseteq H(A)_{\mathbb{Q}_{l}} \subseteq L(A)_{\mathbb{Q}_{l}}=D L_{K}(A)_{\mathbb{Q}_{l}}^{\circ}
$$

By (6.1) we get

$$
\left(G_{l, K, 1}^{a l g}\right)^{\circ}=H(A)_{\mathbb{Q}_{l}}=L(A)_{\mathbb{Q}_{l}}=D L_{K}(A)_{\mathbb{Q}_{l}}^{\circ}
$$

Hence by Remark 4.8, we obtain $\left(G_{l, K}^{a l g}\right)^{\circ}=M T(A)_{\mathbb{Q}_{l}}$. Moreover, since $H(A)$ is closed in $L(A)$, (6.3) gives

$$
H(A)=L(A)
$$

as desired.

Remark 6.4. Recall that $L(A)=D L_{K}(A)^{\circ} \triangleleft D L_{K}^{i d}(A) \triangleleft D L_{K}(A)$. Consider the following epimorphism of groups:

$$
D L_{K}(A) / L(A) \rightarrow D L_{K}(A) / D L_{K}^{i d}(A) \cong G\left(L_{e} / K\right) .
$$

If $A$ satisfies the assumptions of Theorem 6.1, then the epimorphism (6.5) is an isomorphism. This gives an identification $G\left(L_{e} / K\right) \cong A S T_{K}(A) / A S T_{K}(A)^{\circ}$.

We now assemble some cases where the Mumford-Tate and algebraic Sato-Tate conjecture are explained by endomorphisms. We start with some cases with $A$ simple. 
Definition 6.5. Put $g:=\operatorname{dim}(A)$. We say $A$ is of $C M$ type if $D$ contains a commutative $\mathbb{Q}$-subalgebra of dimension $2 g$. If $A$ is simple, then $D L_{\bar{F}}(A)$ is a torus of dimension at most $g$; equality holds if $g \leq 3[\underline{\mathrm{R}}$, Example 3.7]. In this case, $L(A)=D L_{\bar{F}}(A)$.

Theorem 6.6. Let $A / F$ be an abelian variety of $C M$ type. Then $A$ satisfies the conditions of Theorem [6.1, so the algebraic Sato-Tate conjecture holds with $A S T_{K}(A)=D L_{K}(A)$.

Proof. This was shown by Serre using results of Pohlman; see [Se1].

Lemma 6.7. Let $A / F$ be an absolutely simple abelian variety for which the endomorphism algebra $D$ is of type I, II, or III in the Albert classification. Then $C_{D} S p_{(V, \psi)}$ is connected.

Proof. This follows from [BGK1, §7] in the type I and II cases and [BGK2, §5] in the type III case.

Theorem 6.8. Let $A / F$ be an absolutely simple abelian variety of dimension $g$ for which the endomorphism algebra $D$ is of type I, II, or III in the Albert classification. Let $E$ be the center of $D$ and put $e:=[E: \mathbb{Q}], d^{2}=[D: E]$. Assume that $\frac{g}{d e}$ is odd. Then A satisfies the conditions of Theorem [6.1, so the algebraic Sato-Tate conjecture holds with $A S T_{K}(A)=D L_{K}(A)$.

Proof. Recall that $M T(A),\left(G_{l, K}^{a l g}\right)^{\circ},\left(G_{l, K, 1}^{a l g}\right)^{\circ}$ are independent of the field extension $K / F$. We may thus take $K$ large enough that $G_{l, K}^{a l g}$ is connected. In this case, conditions 1 and 2 of Theorem 6.1 are established in BGK1 (in the type I and II case) and BGK2] (in the type III case). Condition 3 holds by Lemma 6.7 .

One can also show that the Mumford-Tate conjecture and the algebraic Sate-Tate conjecture are explained by endomorphisms for all abelian varieties of dimension at most 3. This result is the starting point of the analysis of the Sato-Tate conjecture for abelian surfaces given in [?]; the corresponding analysis for threefolds has not yet been carried out.

Lemma 6.9. For $i=1,2$, let $A_{i} / F$ be an abelian variety satisfying the MumfordTate conjecture. Suppose that over $\bar{F}, A_{1}$ has no factors of type IV while $A_{2}$ either is of CM type or has no factors of type $I V$. Suppose in addition over $\bar{F}$, there is no nontrivial homomorphism from $A_{1}$ to $A_{2}$. Then $A=A_{1} \times A_{2}$ also satisfies the Mumford-Tate conjecture and $H(A)=H\left(A_{1}\right) \times H\left(A_{2}\right)$.

Proof. Let $G, G_{1}, G_{2}$ be the groups $\left(G_{l, F, 1}^{a l g}\right)^{\circ}$ associated to $A, A_{1}, A_{2}$. By hypothesis, we have $G_{1}=H\left(A_{1}\right)_{\mathbb{Q}_{l}}$ and $G_{2}=H\left(A_{2}\right)_{\mathbb{Q}_{l}}$. Since $A_{1}$ has no factors of type IV, $G_{1}$ is semisimple [T, Lemma 1.4]. Similarly, if $A_{2}$ has no factors of type IV, then $G_{2}$ is semisimple; if instead $A_{2}$ is of CM type, then $G_{2}$ is a torus.

There is a natural inclusion $G \subseteq G_{1} \times G_{2}$ with the property that the induced projection maps $\pi_{1}: G \rightarrow G_{1}$ and $\pi_{2}: G \rightarrow G_{2}$ are surjective. Since $G$ is reductive, we can write $G$ as an almost direct product $G^{\prime} \cdot T$ with $G^{\prime}$ semisimple and $T$ a torus.

In case $A_{2}$ is of CM type, we argue following Imai [1]. In this case, $p\left(G^{\prime}\right)=$ $p([G, G])=[p(G), p(G)]=G_{1}$ because $G_{1}$ is semisimple, while $q(T)=q(G)=G_{2}$ because $G^{\prime}$ has no nontrivial character. Hence $\operatorname{dim}(G) \geq \operatorname{dim}\left(G_{1} \times G_{2}\right)$, forcing $G=G_{1} \times G_{2}$. By the same reasoning, $H\left(A_{1}\right)=H\left(A_{1}\right) \times H\left(A_{2}\right)$. 
In case $A_{2}$ has no factors of type IV, then an argument of Hazama Ha, Proposition 1.8] shows that $H(A)=H\left(A_{1}\right) \times H\left(A_{2}\right)$. The same argument implies that if $G \neq G_{1} \times G_{2}$, there is a nonzero $G$-equivariant homomorphism $V_{l}\left(A_{1}\right) \rightarrow V_{l}\left(A_{2}\right)$. However, the latter would imply the existence of a nonzero homomorphism $A_{1} \rightarrow A_{2}$ of abelian varieties over $\bar{F}$, by virtue of Faltings's proof of the Tate conjecture for abelian varieties over number fields $[\mathrm{F}$.

In both cases, we conclude $G=H(A)_{\mathbb{Q}_{l}}=H\left(A_{1}\right)_{\mathbb{Q}_{l}} \times H\left(A_{2}\right)_{\mathbb{Q}_{l}}$, as desired.

Theorem 6.10. Let $A / F$ be an abelian variety of dimension at most 3 . Then A satisfies the conditions of Theorem 6.1, so the Mumford-Tate conjecture holds with $H(A)=L(A)$, and the algebraic Sato-Tate conjecture holds with $A S T_{K}(A)=$ $D L_{K}(A)$.

Proof. Since we are free to enlarge $F$, we may assume that all simple factors of $A$ are absolutely simple. We may invoke $\underline{\mathrm{MZ}}$, Theorem 0.1$]$ to establish condition 1 of Theorem 6.1 in all cases we are considering, so we focus on conditions 2 and 3.

We start with the case where $A$ is simple. If $A$ is a CM elliptic curve, it is straightforward to check condition 2 (or one can apply Theorem 6.6) and condition 3 holds because $D L_{\bar{F}}(A)$ is a torus. If $A$ is a non-CM elliptic curve, condition 2 follows from Serre's open image theorem or from Theorem 6.8 and condition 3 holds because $D L_{\bar{F}}(A)=S L_{2}$. In the other cases, the dimension is prime, so we may deduce condition 2 from [Ch, Concluding Remark] and condition 3 from a theorem of Tankeev [MZ, Theorem 2.7].

We next consider the case where $A$ is not simple. By Theorem 5.3, condition 3 for $A$ reduces to the corresponding condition for each of the simple factors of $A$. We thus need only check condition 2 . In case $A$ is isogenous to a product of elliptic curves, this is a result of Imai $[\mathbf{I}]$. The only other possibility is that $A$ is isogenous to the product of an elliptic curve $E$ with a simple abelian surface $B$; this case may be treated using Lemma 6.9.

Remark 6.11. As noted earlier, Theorem6.10 cannot be extended to dimension 4, due in part to Mumford's examples of absolutely simple abelian fourfolds for which the Hodge conjecture is not explained by endomorphisms. However, there are also some nonsimple examples with the same property. For a complete classification of Hodge groups for abelian varieties of dimension at most 5, see [MZ].

\section{Category of motives for absolute Hodge Cycles}

Even for abelian varieties for which the Mumford-Tate conjecture is not explained by endomorphisms, one can infer from Serre $\mathrm{Se} 4$ a proposed construction of the general algebraic Sato-Tate group in terms of motivic Galois groups and absolute2 Hodge cycles [D1, D2, DM]. In the remainder of the paper, we make the $\ell$-adic interpretation of this construction explicit, and show that if a suitably motivic form of the Mumford-Tate conjecture holds for a particular abelian variety, then the algebraic Sato-Tate conjecture holds as well.

\footnotetext{
${ }^{2}$ Since we consider only the motives associated to abelian varieties, the adjective absolute is rendered unnecessary by Deligne's theorem equating Hodge cycles with absolute Hodge cycles [D1]. However, we prefer to speak of absolute Hodge cycles in order to emphasize the action of Galois on them, which is crucial for our construction of the disconnected part of the algebraic Sato-Tate group.
} 
To begin with, we recall some terminology and set some notation concerning Tannakian categories. Let $\mathcal{C}$ be a unital, $\mathbb{Q}$-linear Tannakian category equipped (as in $\left[\mathrm{DM}\right.$, Definition 2.19]) with a $\mathbb{Q}$-linear, faithful tensor functor $\omega: \mathcal{C} \rightarrow \mathrm{Vec}_{\mathbb{Q}}$, called the fiber functor of $\mathcal{C}$.

Definition 7.1. For $X, Y \in \operatorname{obj}(\mathcal{C})$, let $\underline{\operatorname{Hom}}_{\mathcal{C}}(X, Y) \in \operatorname{obj}(\mathcal{C})$ be the object which represents the functor

$$
\mathcal{C}^{\circ} \rightarrow \text { Set, } \quad T \mapsto \operatorname{Hom}_{\mathcal{C}}(T \otimes X, Y) .
$$

That is, for every $T \in \operatorname{obj}(\mathcal{C})$ there is a natural bijection

$$
\operatorname{Hom}_{\mathcal{C}}(X \otimes T, Y) \cong \operatorname{Hom}_{\mathcal{C}}\left(T, \underline{\operatorname{Hom}}_{\mathcal{C}}(X, Y)\right) \text {. }
$$

One puts $X^{\vee}:=\underline{\operatorname{Hom}}_{\mathcal{C}}(X, \underline{1})$ and one gets $\underline{\operatorname{Hom}}_{\mathcal{C}}(X, Y) \cong X^{\vee} \otimes Y$.

Proposition 7.2. ([DM, Proposition 1.9]) For a functor $F: \mathcal{C} \rightarrow \mathcal{C}^{\prime}$ of unital Tannakian categories and $X, Y \in \mathrm{obj}(\mathcal{C})$, there is a natural isomorphism

$$
F\left(\underline{\operatorname{Hom}}_{\mathcal{C}}(X, Y)\right) \cong \underline{\operatorname{Hom}}_{\mathcal{C}^{\prime}}(F(X), F(Y)) .
$$

Let us consider now the case of motives.

Definition 7.3. Let $K$ be a number field and let $\mathcal{M}_{K}$ be the motivic category for absolute Hodge cycles: $\operatorname{Hom}_{\mathcal{M}_{K}}(M, N):=\operatorname{Mor}_{A H}^{0}(X, Y)$ (see [DM]). Define $\mathcal{M}_{\bar{K}}$ similarly. Let $H_{B}$ be the fiber functor given by Betti realization:

$$
H_{B}: \mathcal{M}_{K} \rightarrow \operatorname{Vec}_{\mathbb{Q}} \text {. }
$$

The functor $H_{B}$ factors through the functor

$$
\mathcal{M}_{K} \rightarrow \mathcal{M}_{\bar{K}}, \quad M \mapsto \bar{M}:=M \otimes \bar{K} .
$$

Definition 7.4. Let $\left\{X_{i}\right\}$ be a family of varieties over $K$ and let $\mathcal{C} \subset \mathcal{M}_{K}$ be the full Tannakian subcategory generated by $\left\{h\left(X_{i}\right)\right\}$. Let $\overline{\mathcal{C}}$ be the smallest full Tannakian subcategory of $\mathcal{M}_{\bar{K}}$ containing the image of $\mathcal{C}$ via the functor (7.3).

The category $\overline{\mathcal{C}}$ is the full Tannakian subcategory of $\mathcal{M}_{\bar{K}}$ generated by $\left\{h\left(\overline{X_{i}}\right)\right\}$, c.f. [DM, pp. 215-216]. For $M, N \in \operatorname{obj}(\mathcal{C})$ the natural map

$$
\operatorname{Hom}_{\bar{C}}(\bar{M}, \bar{N}) \times \bar{M} \rightarrow \bar{N}
$$

gives the natural map

$$
\operatorname{Hom}_{\overline{\mathcal{C}}}(\bar{M}, \bar{N}) \rightarrow \underline{\operatorname{Hom}}_{\overline{\mathcal{C}}}(\bar{M}, \bar{N})
$$

where $\operatorname{Hom}_{\overline{\mathcal{C}}}(\bar{M}, \bar{N})=\operatorname{Hom}_{\mathcal{M}_{K}}(\bar{M}, \bar{N})$ is a finite dimensional vector space which is also a discrete $G_{K}$-module, so it can be considered as an Artin motive in $\mathcal{M}_{K}$. Moreover by (7.1) we get $\underline{\operatorname{Hom}}_{\overline{\mathcal{C}}}(\bar{M}, \bar{N}) \cong \underline{\operatorname{Hom}}_{\mathcal{M}_{\bar{K}}}(\bar{M}, \bar{N})$. Hence the map (7.5) is naturally isomorphic to the map

$$
\operatorname{Hom}_{\mathcal{M}_{\bar{K}}}(\bar{M}, \bar{N}) \rightarrow \underline{\operatorname{Hom}}_{\mathcal{M}_{\bar{K}}}(\bar{M}, \bar{N}) .
$$

Let $L / K$ be a field extension with $L \subset \bar{K}$. For any variety $X / L$ of pure dimension $m$, one sets $h(X)^{\vee}:=h(X)(m)$, which extends to the contravariant functor

$$
\mathcal{M}_{L} \rightarrow \mathcal{M}_{L}, \quad M \mapsto M^{\vee}
$$

and one sets (see [DM, p. 205])

$$
\underline{\operatorname{Hom}}_{\mathcal{M}_{L}}(M, N):=M^{\vee} \otimes N \text {. }
$$


Since the map (7.4) commutes with the $G_{K}$-action, using (7.7) the maps (7.5) and (7.6) also commute with the $G_{K}$-action.

Proposition 7.5. There is a natural morphism of motives in $\mathcal{M}_{K}$ :

$$
\operatorname{Hom}_{\mathcal{M}_{\bar{K}}}(\bar{M}, \bar{N}) \rightarrow \underline{\operatorname{Hom}}_{\mathcal{C}}(M, N),
$$

which is an embedding of motives.

Proof. The existance of the morphism (7.8) follows from the definition of morphisms for the category of motives for absolute Hodge cycles. Let $\underline{1}$ be the unit of $\mathcal{M}_{\bar{K}}$. Applying $\operatorname{Hom}_{\mathcal{M}_{\bar{K}}}(\underline{1}$, . ) to the map (17.6) one immediately gets the identity map $\operatorname{Hom}_{\mathcal{M}_{\bar{K}}}(\bar{M}, \bar{N}) \rightarrow \operatorname{Hom}_{\mathcal{M}_{\bar{K}}}(\bar{M}, \bar{N})$. This shows that the map (7.8) is an embedding of motives.

\section{Motivic Mumford-Tate and Sato-Tate groups}

Using the Tannakian formalism, we now define a motivic Sato-Tate group by reprising the setup from 33 ,

Definition 8.1. Let $G_{\mathcal{M}_{K}}:=\operatorname{Aut}^{\otimes}\left(H_{B}\right)$ and let $\mathcal{M}_{K}(A)$ be the smallest Tannakian subcategory of $\mathcal{M}_{K}$ containing $A$. Let $H_{B} \mid \mathcal{M}_{K}(A)$ be the restriction of $H_{B}$ to the subcategory $\mathcal{M}_{K}(A)$. Put $G_{\mathcal{M}_{K}(A)}:=\operatorname{Aut}^{\otimes}\left(H_{B} \mid \mathcal{M}_{K}(A)\right)$.

The algebraic groups $G_{\mathcal{M}_{K}(A)}$ are reductive but not necessarily connected (see $\left[\right.$ Se4, p. 379]). Observe that the ring of endomorphisms $D:=\operatorname{End}_{\bar{K}}(A) \otimes_{\mathbb{Z}} \mathbb{Q}$ is a discrete $G_{K}$-module which is also a finite dimensional $\mathbb{Q}$-vector space. Moreover (see [DM, p. 213]),

$$
\operatorname{End}_{\bar{K}}(A) \otimes_{\mathbb{Z}} \mathbb{Q}=\operatorname{End}_{\bar{K}}(\bar{A}) \otimes_{\mathbb{Z}} \mathbb{Q}=\operatorname{End}_{\mathcal{M}_{\bar{K}}}\left(h^{1}(\bar{A})\right) .
$$

We may thus consider $D$ as an Artin motive, as follows. Recall that $\mathcal{M}_{K}^{0}$ is equivalent to $\operatorname{Rep}_{\mathbb{Q}}\left(G_{K}\right)$, the category of finite dimensional $\mathbb{Q}$-vector spaces with continuous actions of $G_{K}$.

Definition 8.2. Let $h^{0}(D)$ denote the Artin motive corresponding to $D$. Let $\mathcal{M}_{K}^{0}(D)$ be the smallest Tannakian subcategory of $\mathcal{M}_{K}^{0}$ containing $h^{0}(D)$ and put $G_{\mathcal{M}_{K}^{0}(D)}:=\operatorname{Aut}^{\otimes}\left(H_{B}^{0} \mid \mathcal{M}_{K}^{0}(D)\right)$.

By (7.1), Proposition 7.5, and (8.1), we have a natural embedding of motives

$$
h^{0}(D) \subset \text { End }_{\mathcal{M}_{K}(A)}\left(h^{1}(A)\right)=\text { End }_{\mathcal{M}_{K}}\left(h^{1}(A)\right) .
$$

Note that $\underline{\operatorname{End}}_{\mathcal{M}_{K}(A)}\left(h^{1}(A)\right)=h^{1}(A) \otimes h^{1}(A)^{\vee} \in \mathcal{M}_{K}(A)$. Recall that $G_{\mathcal{M}_{K}^{0}} \cong G_{K}$ so we observe that

$$
G_{\mathcal{M}_{K}^{0}(D)} \cong G\left(L_{e} / K\right)
$$

Since $\mathcal{M}_{K}$ is semisimple [DM, Prop. 6.5], the motive $h^{0}(D)$ splits off of End $\underline{\mathcal{M}}_{K}(A)\left(h^{1}(A)\right)$ in $\mathcal{M}_{K}$. Moreover the semisimplicity of $\mathcal{M}_{K}$, together with the observation that $\mathcal{M}_{K}^{0}$ and $\mathcal{M}_{K}(A)$ are full subcategories of $\mathcal{M}_{K}$, shows that the top horizontal and left vertical maps in the following diagram are faithfully flat (see [DM, (2.29)]):

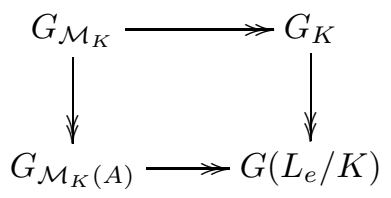


In particular all homomorphisms in (8.3) are surjective.

Since $V:=H_{1}(A(\mathbb{C}), \mathbb{Q})$ admits a $\mathbb{Q}$-rational polarized Hodge structure of weight 1 , we have

$$
G_{\mathcal{M}_{K}(A)} \subset G S p_{(V, \psi)} .
$$

Definition 8.3. Define the following algebraic groups:

$$
\begin{aligned}
G_{\mathcal{M}_{K}(A), 1} & :=G_{\mathcal{M}_{K}(A)} \cap S p_{V} \\
G_{\mathcal{M}_{K}(A), 1}^{\circ} & :=\left(G_{\mathcal{M}_{K}(A)}\right)^{\circ} \cap S p_{V} .
\end{aligned}
$$

Remark 8.4. In [Se4, p. 396] the group $G_{\mathcal{M}_{K}(A), 1}$ is denoted $G_{\mathcal{M}_{K}(A)}^{1}$.

Definition 8.5. For any $\tau \in G\left(L_{e} / K\right)$, put

$$
G S p_{(V, \psi)}^{\tau}:=\left\{g \in G S p_{(V, \psi)}: g \beta g^{-1}=\rho_{e}(\tau)(\beta) \forall \beta \in D\right\} .
$$

We have the following equality:

$$
G S p_{(V, \psi)}=\bigsqcup_{\tau \in G\left(L_{e} / K\right)} G S p_{(V, \psi)}^{\tau} .
$$

Observe that

$$
G S p_{(V, \psi)}^{I d}=C_{D}\left(G S p_{(V, \psi)}\right) .
$$

Remark 8.6. The bottom horizontal arrow in the diagram (8.3) is

$$
G_{\mathcal{M}_{K}(A)} \rightarrow G_{\mathcal{M}_{K}(D)} \cong G\left(L_{e} / K\right) \text {. }
$$

Let $g \in G_{\mathcal{M}_{K}(A)}$ and let $\tau:=\tau(g)$ be the image of $g$ via the map (8.8). Hence for any element $\beta \in D$ considered as an endomorphism of $V$ we have:

$$
g \beta g^{-1}=\rho_{e}(\tau)(\beta) .
$$

Definition 8.7. For any $\tau \in G\left(L_{e} / K\right)$, put

$$
G_{\mathcal{M}_{K}(A)}^{\tau}:=\left\{g \in G_{\mathcal{M}_{K}(A)}: g \beta g^{-1}=\rho_{e}(\tau)(\beta), \quad \forall \beta \in D\right\} .
$$

It follows from (8.9), (8.10), and the surjectivity of (8.8) that

$$
G_{\mathcal{M}_{K}(A)}=\bigsqcup_{\tau \in G\left(L_{e} / K\right)} G_{\mathcal{M}_{K}(A)}^{\tau}
$$

It is clear from (8.4) and (8.5) that

$$
G_{\mathcal{M}_{K}(A)}^{\tau} \subset G S p_{(V, \psi)}^{\tau} .
$$

Hence (8.9) and (8.11) give

$$
\left(G_{\mathcal{M}(A)}\right)^{\circ} \triangleleft G_{\mathcal{M}_{K}(A)}^{I d} \triangleleft G_{\mathcal{M}_{K}(A)} .
$$

The map (8.8) gives the following natural map:

$$
G_{\mathcal{M}_{K}(A), 1} \rightarrow G\left(L_{e} / K\right) .
$$

Definition 8.8. For any $\tau \in G\left(L_{e} / K\right)$ put

$$
G_{\mathcal{M}_{K}(A), 1}^{\tau}:=\left\{g \in G_{\mathcal{M}_{K}(A), 1}: g \beta g^{-1}=\rho_{e}(\tau)(\beta), \quad \forall \beta \in D\right\} .
$$


It follows that there is the following equality

$$
G_{\mathcal{M}_{K}(A), 1}^{\tau}=G_{\mathcal{M}_{K}(A), 1} \cap G_{\mathcal{M}_{K}(A)}^{\tau} .
$$

Hence for $\tau=\tau(g)$, we have

$$
\begin{aligned}
G_{\mathcal{M}_{K}(A), 1}^{\tau} & \subset D L_{K}^{\tau}(A) \\
G_{\mathcal{M}_{K}(A), 1} & \subset D L_{K}(A) .
\end{aligned}
$$

Naturally we also have

$$
\left(G_{\mathcal{M}(A), 1}\right)^{\circ} \triangleleft G_{\mathcal{M}_{K}(A), 1}^{I d} \triangleleft G_{\mathcal{M}_{K}(A), 1}
$$

Hence by (8.16) we get

$$
G_{\mathcal{M}(A), 1} / G_{\mathcal{M}_{K}(A), 1}^{I d} \subset G_{\mathcal{M}(A)} / G_{\mathcal{M}_{K}(A)}^{I d} .
$$

We have the following analogue of Theorem 3.4

Theorem 8.9. Assume that $G_{\mathcal{M}_{K}(A), 1}^{\circ}$ is connected. Then the following map is an isomorphism:

$$
i_{M}: G_{\mathcal{M}_{K}(A), 1} /\left(G_{\mathcal{M}_{K}(A), 1}\right)^{\circ} \stackrel{\cong}{\longrightarrow} G_{\mathcal{M}_{K}(A)} /\left(G_{\mathcal{M}_{K}(A)}\right)^{\circ} .
$$

Proof. Consider the following diagram, in which we write $\mathcal{M}(A)$ for $\mathcal{M}_{K}(A)$ to make the notation simpler.

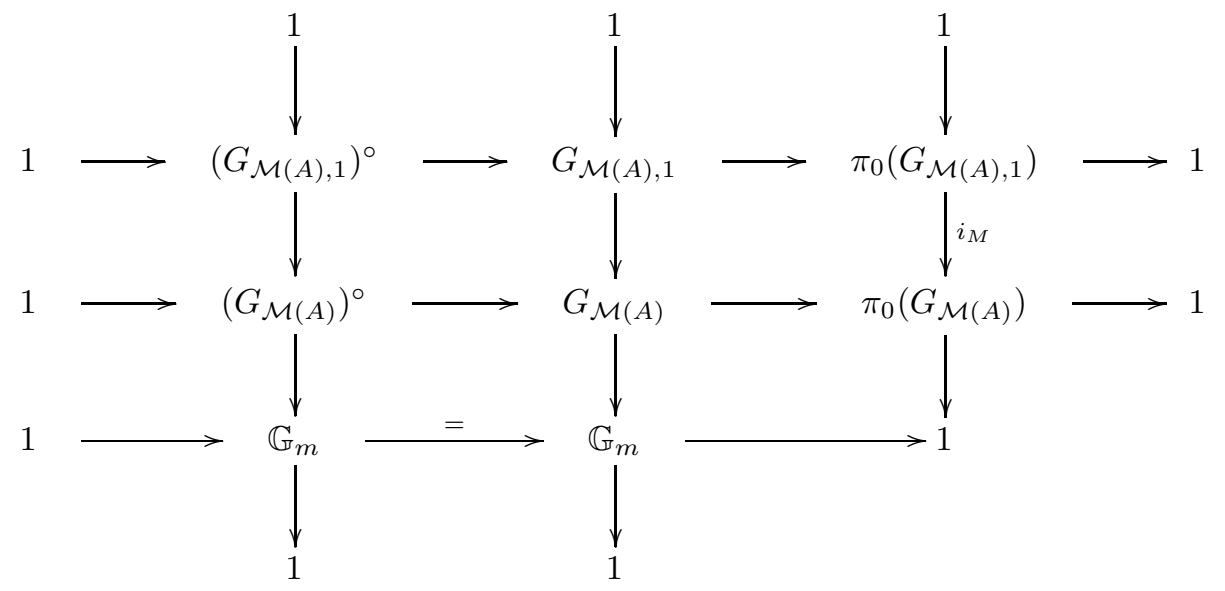

By definition the rows are exact and the middle column is exact. Hence the map $i_{M}$ is surjective. Since $G_{\mathcal{M}_{K}(A), 1}^{\circ}$ has the same dimension as $G_{\mathcal{M}_{K}(A), 1}$ and by assumption $G_{\mathcal{M}_{K}(A), 1}^{\circ}$ is connected, we then have $G_{\mathcal{M}_{K}(A), 1}^{\circ}=\left(G_{\mathcal{M}_{K}(A), 1}\right)^{\circ}$. Hence the left column is also exact. This shows that $i_{M}$ is an isomorphism.

Remark 8.10. Since $G_{\mathcal{M}(A)}$ is reductive, the middle vertical column of the diagram of the proof of Theorem 8.9) shows that $G_{\mathcal{M}(A), 1}$ is also reductive. 
Corollary 8.11. Assume that $G_{\mathcal{M}_{K}(A), 1}^{\circ}$ is connected. Then there are natural isomorphisms

$$
\begin{aligned}
G_{\mathcal{M}_{K}(A), 1} / G_{\mathcal{M}_{K}(A), 1}^{I d} & \cong \\
G_{\mathcal{M}_{K}(A), 1}^{I d} /\left(G_{\mathcal{M}_{K}(A), 1}\right)^{\circ} & \stackrel{\cong}{\longrightarrow} G_{\mathcal{M}_{K}(A)}^{I d} / G_{\mathcal{M}_{K}(A)}^{I d}, \\
\left.G_{\mathcal{M}_{K}(A), 1} / G_{\mathcal{M}_{K}(A)}^{I d}\right)^{\circ}, & \stackrel{\cong}{\longrightarrow} D L_{K}(A) / D L_{K}(A)^{I d} \stackrel{\cong}{\longrightarrow} G\left(L_{e} / K\right) .
\end{aligned}
$$

In particular the natural map 8.14 is surjective.

Proof. This follows from (8.13), (8.19), (8.20), the surjectivity of (8.8), and Theorem 8.9

Remark 8.12. The $l$-adic representation $\rho_{l}: G_{K} \rightarrow G_{\mathcal{M}_{K}(A)}\left(\mathbb{Q}_{l}\right)$ associated with $A$ factors through $G_{\mathcal{M}_{K}(A)}\left(\mathbb{Q}_{l}\right)$ (see $[\mathrm{Se} 4$, p. 386]). Hence

$$
G_{l, K}^{a l g} \subset G_{\mathcal{M}_{K}(A)_{\mathbb{Q}_{l}}}
$$

where $G_{\mathcal{M}_{K}(A)_{\mathbb{Q}_{l}}}:=G_{\mathcal{M}_{K}(A)} \otimes_{\mathbb{Q}} \mathbb{Q}_{l}$. By the commutativity of the diagram

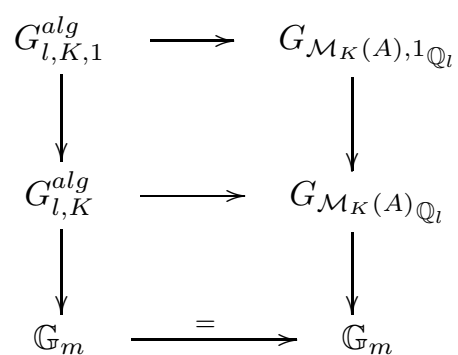

it follows that

$$
G_{l, K, 1}^{a l g} \subset G_{\mathcal{M}_{K}(A), 1_{\mathbb{Q}_{l}}}
$$

Definition 8.13. The algebraic groups

$$
\begin{aligned}
\operatorname{MMT}_{K}(A) & :=G_{\mathcal{M}_{K}(A)} \\
M_{S T}(A) & :=G_{\mathcal{M}_{K}(A), 1}
\end{aligned}
$$

will be called the motivic Mumford-Tate group and motivic Sato-Tate group for A.

Conjecture 8.14. (Motivic Mumford-Tate) For any prime number l,

$$
G_{l, K}^{a l g}=M M T_{K}(A)_{\mathbb{Q}_{l}} .
$$

By the diagram above, Conjecture 8.14 is equivalent to the following.

Conjecture 8.15. (Motivic Sato-Tate) For any prime number l,

$$
G_{l, K, 1}^{a l g}=M S T_{K}(A)_{\mathbb{Q}_{l}} .
$$

Remark 8.16. Conjecture 8.14 is equivalent to the conjunction of the following equalities:

$$
\begin{aligned}
\left(G_{l, K}^{a l g}\right)^{\circ} & =\left(M M T_{K}(A)_{\mathbb{Q}_{l}}\right)^{\circ} \\
\pi_{0}\left(G_{l, K}^{a l g}\right) & =\pi_{0}\left(M M T_{K}(A)_{\mathbb{Q}_{l}}\right) .
\end{aligned}
$$


Similarly, Conjecture 8.15 is equivalent to the conjunction of the following equalities:

$$
\begin{aligned}
\left(G_{l, K, 1}^{a l g}\right)^{\circ} & =\left(M S T_{K}(A)_{\mathbb{Q}_{l}}\right)^{\circ} \\
\pi_{0}\left(G_{l, K, 1}^{a l g}\right) & =\pi_{0}\left(M S T_{K}(A)_{\mathbb{Q}_{l}}\right) .
\end{aligned}
$$

Theorem 8.17. We have the following results.

1. An isogeny $\phi: A_{1} \rightarrow A_{2}$ of abelian varieties over a number field $K$ induces isomorphisms $G_{\mathcal{M}_{K}\left(A_{1}\right)} \cong G_{\mathcal{M}_{K}\left(A_{2}\right)}$ and $G_{\mathcal{M}_{K}\left(A_{1}\right), 1} \cong G_{\mathcal{M}_{K}\left(A_{2}\right), 1}$.

2. If $A$ is an abelian variety over a number field $K$, then for every positive integer $s, \quad G_{\mathcal{M}_{K}\left(A^{s}\right)} \cong G_{\mathcal{M}_{K}(A)}$ and $G_{\mathcal{M}_{K}\left(A^{s}\right), 1} \cong G_{\mathcal{M}_{K}(A), 1}$.

Proof. 1. Observe that an isogeny $\phi$ induces a natural isomorphism $V\left(A_{1}\right) \cong V\left(A_{2}\right)$ and a natural equivalence of categories $\mathcal{M}_{K}\left(A_{1}\right) \cong \mathcal{M}_{K}\left(A_{2}\right)$. This leads to a natural equivalence of fiber functors $H_{B}\left|\mathcal{M}_{K}\left(A_{1}\right) \cong H_{B}\right| \mathcal{M}_{K}\left(A_{2}\right)$.

2. By the Künneth formula, there is an isomorphism of motives $h^{1}\left(A^{s}\right) \cong h^{1}(A)^{s}$. In addition for any abelian variety $B$ over $K$ we have $h(B)=\wedge h^{1}(B)$. This shows that the diagonal map $\Delta: A \rightarrow A^{s}$ induces a natural equivalence of categories $\mathcal{M}_{K}(A) \cong \mathcal{M}_{K}\left(A^{s}\right)$. Moreover there is a natural isomorphism $V\left(A^{s}\right) \cong V(A)^{s}$. Hence there is a natural equivalence of fiber functors $H_{B}\left|\mathcal{M}_{K}\left(A^{s}\right) \cong H_{B}\right| \mathcal{M}_{K}(A)$.

Corollary 8.18. Let $A / K$ be an abelian variety such that the motivic MumfordTate and motivic Sato-Tate conjectures hold for A. Then the motivic Mumford-Tate and motivic Sato-Tate conjectures hold for $A^{s}$ for every positive integer $s$.

Proof. This follows from Theorems 4.10 and 8.17

\section{The algebraic Sato-Tate group}

To conclude, we introduce a candidate for the algebraic Sato-Tate group for an arbitrary abelian variety.

Remark 9.1. One observes (cf. [Se4, p. 379]) that

$$
M T(A) \subset\left(G_{\mathcal{M}_{K}(A)}\right)^{\circ}
$$

and it was proven by Deligne that

$$
\left(G_{l, K}^{a l g}\right)^{\circ} \subset M T(A)_{\mathbb{Q}_{l}} .
$$

It follows by (8.17), (8.19), and (9.1) that for every abelian variety $A / K$ there is a sequence of natural containments

$$
H(A) \subset\left(G_{\mathcal{M}_{K}(A), 1}\right)^{\circ} \subset G_{\mathcal{M}_{K}(A), 1}^{I d} \subset C_{D}\left(S p_{(V, \psi)}\right) .
$$

In the same way, it follows by (8.7), (8.12), (8.13), and (9.1) that for every abelian variety $A / K$ there is a sequence of natural containments

$$
M T(A) \subset\left(G_{\mathcal{M}_{K}(A)}\right)^{\circ} \subset G_{\mathcal{M}_{K}(A)}^{I d} \subset C_{D}\left(G S p_{(V, \psi)}\right) .
$$

We observe that the equality

$$
H(A)=C_{D}\left(S p_{(V, \psi)}\right)
$$

is equivalent to the following equality:

$$
M T(A)=C_{D}\left(G S p_{(V, \psi)}\right) .
$$


Remark 9.2. It is conjectured [Se4, sec. 3.4] that $M T(A)=M M T_{K}(A)^{\circ}=$ $\left(G_{\mathcal{M}_{K}(A)}\right)^{\circ}$, and in [Se4, p. 380] J.-P. Serre gave examples where this conjecture holds. Theorems 9.3 and 9.4 below gives more evidence for this conjecture.

Theorem 9.3. Let $A / F$ be an absolutely simple abelian variety of dimension $g$ for which the endomorphism algebra D is of type I, II, or III in the Albert classification. Let $E$ be the center of $D$ and put $e:=[E: \mathbb{Q}], d^{2}=[D: E]$. Assume that $\frac{g}{d e}$ is odd. Then

$$
\begin{aligned}
H(A) & =M S T_{K}(A)^{\circ}=\left(G_{\mathcal{M}_{K}(A), 1}\right)^{\circ}=C_{D}\left(S p_{(V, \psi)}\right), \\
M T(A) & =M M T_{K}(A)^{\circ}=\left(G_{\mathcal{M}_{K}(A)}\right)^{\circ}=C_{D}\left(G S p_{(V, \psi)}\right) .
\end{aligned}
$$

Proof. By [BGK1, Cor. 7.19] and [BGK2, Cor. 5.19], for abelian varieties satisfying the assumptions of the theorem, the equality (9.5) follows.

Theorem 9.4. Let $A / F$ be an abelian variety of dimension at most 3 . Then the equalities (9.7) and (9.8) hold also for $A$.

Proof. For abelian varieties of dimension at most 3 the equality (9.5) holds by Theorem 6.10

Remark 9.5. To obtain $G_{l, K}^{a l g}$ as an extension of scalars to $\mathbb{Q}_{l}$ of an expected algebraic Sato-Tate group defined over $\mathbb{Q}$, the assumption in the following definition is natural in view of (9.1), (9.2), and Theorem 8.9.

Definition 9.6. Assume that $M T(A)=M M T_{K}(A)^{\circ}$. Then the algebraic SatoTate group $A S T_{K}(A)$ is defined as follows:

$$
A S T_{K}(A):=\operatorname{MST}_{K}(A)=G_{\mathcal{M}_{K}(A), 1} .
$$

Every maximal compact subgroup of $A S T_{K}(A)(\mathbb{C})$ will be called a Sato-Tate group associated with $A / K$ and denoted $S T_{K}(A)$.

Corollary 9.7. Let $A / K$ be an abelian variety such that $M T(A)=M M T_{K}(A)^{\circ}$ and the algebraic Sato-Tate conjecture holds for A. Then the algebraic Sato-Tate conjecture holds for $A^{s}$ for every positive integer $s$.

Proof. It follows immediately from Corollary 8.18

We now prove that the group $\operatorname{AST}_{K}(A)$ satisfies the expected properties stated in Remark 2.5,

Theorem 9.8. Assume that $M T(A)=M M T_{K}(A)^{\circ}$. Then the group $A S T_{K}(A)$ is reductive and:

$$
\begin{aligned}
A S T_{K}(A) & \subset D L_{K}(A), \\
A S T_{K}(A)^{0} & =H(A), \\
\pi_{0}\left(A S T_{K}(A)\right) & =\pi_{0}\left(M M T_{K}(A)\right), \\
\pi_{0}\left(A S T_{K}(A)\right) & =\pi_{0}\left(S T_{K}(A)\right) .
\end{aligned}
$$

In addition, there are the following exact sequences:

$$
\begin{gathered}
\quad 0 \rightarrow H(A) \rightarrow A S T_{K}(A) \rightarrow \pi_{0}\left(A S T_{K}(A)\right) \rightarrow 0 \\
0 \rightarrow \pi_{0}\left(G_{\mathcal{M}_{K}(A), 1}^{I d}\right) \rightarrow \pi_{0}\left(A S T_{K}(A)\right) \rightarrow G\left(L_{e} / K\right) \rightarrow 0 .
\end{gathered}
$$


Proof. The group $A S T_{K}(A)$ is reductive by Remark 8.10, and (9.10) follows by (8.18). Equality (9.13) follows since $A S T_{K}(A)^{\circ}(\mathbb{C})$ is a connected complex Lie group and any maximal compact subgroup of a connected complex Lie group is a connected real Lie group. Assuming that $M T(A)=\left(G_{\mathcal{M}_{K}(A)}\right)^{\circ}$, we have

$$
G_{\mathcal{M}_{K}(A), 1}^{\circ}=\left(G_{\mathcal{M}_{K}(A)}\right)^{\circ} \cap S p_{(V, \psi)}=M T(A) \cap S p_{(V, \psi)}=H(A),
$$

so $G_{\mathcal{M}_{K}(A), 1}^{\circ}$ is connected. Hence

$$
A S T_{K}(A)^{0}=\left(G_{\mathcal{M}_{K}(A), 1}\right)^{\circ}=G_{\mathcal{M}_{K}(A), 1}^{\circ},
$$

so (9.11) follows. Hence the equality (9.12) and the exact sequence (9.14) may be extracted from the top row of the diagram from the proof of Theorem 8.9. The exactness of the sequence (9.15) follows immediately from Corollary 8.11.

Remark 9.9. The containments (9.3) give some approximation for $\pi_{0}\left(G_{\mathcal{M}_{K}(A), 1}^{I d}\right)$. Moreover, under the assumption $M T(A)=\left(G_{\mathcal{M}_{K}(A)}\right)^{\circ}$ of Definition 9.6, we have the following commutative diagrams:

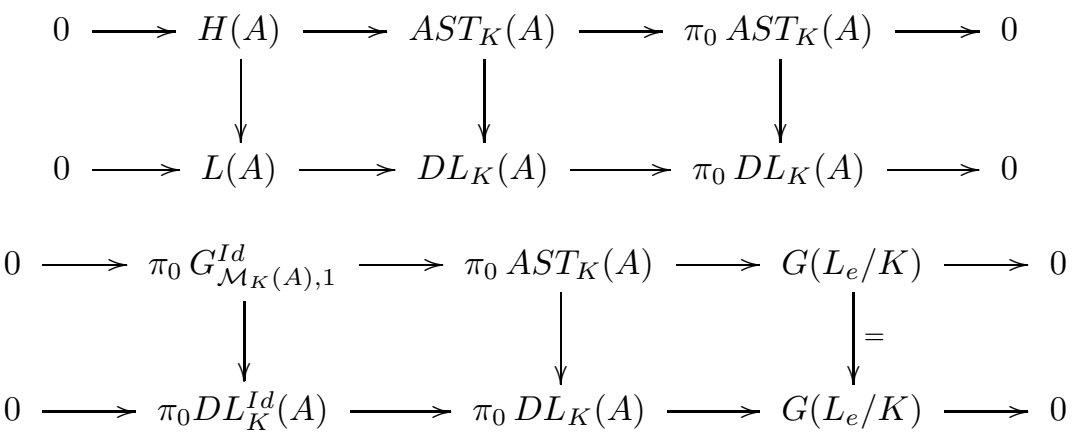

Corollary 9.10. Asume that $H(A)=C_{D}\left(S p_{(V, \psi)}\right)$. Then

$$
A S T_{K}(A)=D L_{K}(A) .
$$

Proof. It follows by the assumption and (9.3) that $\pi_{0}\left(G_{\mathcal{M}_{K}(A), 1}^{I d}\right)=\pi_{0}\left(D L_{K}^{I d}(A)\right)=$ 1. Hence the middle vertical arrow in the diagram (9.19), which is the right vertical arrow in the diagram (9.18), is an isomorphism. Since $L(A):=\left(C_{D} S p_{(V, \psi)}\right)^{\circ}$, by our assumption we have $H(A)=L(A)$. Hence the left vertical arrow in the diagram (9.18) is an isomorphism, so the middle vertical arrow in the diagram (9.18) is an isomorphism.

Corollary 9.11. If $H(A)=C_{D}\left(S p_{(V, \psi)}\right)$ and the Mumford-Tate conjecture holds for $A$ then the algebraic Sato-Tate conjecture holds for $A S T_{K}(A):=G_{\mathcal{M}_{K}(A), 1}$, namely

$$
G_{l, K, 1}^{a l g}=A S T_{K}(A)_{\mathbb{Q}_{l}}
$$

Proof. By (8.25) and Corollary 9.10, we get

$$
G_{l, K, 1}^{a l g} \subset A S T_{K}(A)_{\mathbb{Q}_{l}}=D L_{K}(A)_{\mathbb{Q}_{l}} .
$$

By the assumption $H(A)=D L_{L_{e}}(A)$, the equivalence of (5.11) and (5.12) show that we need to prove that $\left(G_{l, K, 1}^{a l g}\right)^{\circ}=H(A)_{\mathbb{Q}_{l}}$. However, this is equivalent to the Mumford-Tate conjecture by Remark 4.8 
Remark 9.12. The results of this and the previous chapter, in particular Theorem 9.8 and Corollaries 9.10 and 9.11, show that the group $A S T_{K}(A)$ from Definition 9.6 is a natural candidate for the algebraic Sato-Tate group for the abelian variety $A$.

Remark 9.13. For absolutely simple $A / F$ for which the endomorphism algebra $D$ is of type I, II, or III in the Albert classification with $\frac{g}{d e}$ odd and for $A / F$ of dimension at most 3 we explicitely determined the motivic Mumford-Tate and motivic Sato-Tate groups (see (9.12) of Theorem 9.8. Corollary 9.10, Theorems 9.3 and (9.4).

\section{REFERENCES}

[BGK1] G. Banaszak, W. Gajda, P. Krason, On the image of $l$-adic Galois representations for abelian varieties of type I and II, Doc. Math., Extra Volume, John Coates Sixtieth Birthday (2006), 35-75.

[BGK2] G. Banaszak, W. Gajda, P. Krasoń, On the image of Galois l-adic representations for abelian varieties of type III, Tohoku Math. J., 62, No. 2 (2010), 163-189.

[BLGG] T. Barnet-Lamb, D. Geraghty, and T. Gee, The Sato-Tate conjecture for Hilbert modular forms, J. Amer. Math. Soc. 24 (2011), 411-469.

[Bo] F.A. Bogomolov, Sur l'algébricité des représentations l-adiques, C. R. Acad. Sci. Paris Sér. A-B 290, (1980), A701-A703.

[Ch] W. Chi, On the $\ell$-adic representations attached to simple abelian varieties of type IV, Bull. Austral. Math. Soc. 44 (1991), 71-78.

[D1] P. Deligne, Théorie de Hodge III, Publ. Math. IHÉS 44 (1974), 5-77.

[D2] P. Deligne, Hodge cycles on abelian varieties, in Hodge Cycles, Motives, and Shimura Varieties, Lecture Notes in Math. 900, Springer, 1982, 9-100.

[DM] P. Deligne, J.S. Milne, Tannakian categories, Lecture Notes in Math. 900, (1982), 100-228.

[F] G. Faltings, Endlichkeitssätze für abelsche Varietäten über Zalhkörpern, Inv. Math. 73 (1983), 349-366.

[FKRS12] F. Fité, K.S. Kedlaya, V. Rotger, A.V. Sutherland, Sato-Tate distributions and Galois endomorphism modules in genus 2, Compos. Math. 148 (2012), 1390-1442.

[G] B. Gordon, A survey of the Hodge conjecture for abelian varieties, Appendix B in J. Lewis, A Survey of the Hodge Conjecture, Amer. Math. Soc., 1999, 297-356.

[Ha] F. Hazama, Algebraic cycles on nonsimple abelian varieties, Duke. Math. J. 58 (1989), $31-37$.

[I] H. Imai, On the Hodge groups of some abelian varieties, Kodai Math. Sem. Rep. 27 (1976), 367-372.

[Md] D. Mumford, Abelian Varieties, Tata Institute, Oxford University Press, 1988.

[Mi] J.S. Milne, Abelian varieties, in Arithmetic Geometry, Springer-Verlag, 1986.

[Mo] F. Momose, On the $l$-adic representations attached to modular forms, J. Fac. Sci. Univ. Tokyo 28 (1981), 89-109.

[MZ] B.J.J. Moonen and Yu.G. Zarhin, Hodge classes on abelian varieties of low dimension, Math. Ann. 315 (1999), 711-733.

[My] V.K. Murty, Exceptional Hodge classes on certain abelian varieties, Math. Ann. 268 (1984), 197-206.

[R] K.A. Ribet, Division points of abelian varieties with complex multiplication, Mém. Soc. Math. France 2e Ser. 2 (1980), 75-94.

[Se1] J.-P. Serre, Representations l-adiques, in S. Iyanaga (ed.), Algebraic Number Theory, Japan Society for the Promotion of Science, Kyoto University Press, 1977, 177-193.

[Se2] J.-P. Serre, Letter to K. Ribet, Jan. 29, 1981, in CEuvres. Collected Papers. IV. 19851998, Springer-Verlag, Berlin, 2000.

[Se3] J.-P. Serre, Course at the Collège de France, 1984-85, in Euvres. Collected Papers. IV. 1985-1998, Springer-Verlag, Berlin, 2000. 
[Se4] J.-P. Serre, Propriétés conjecturales des groupes de Galois motiviques et des représentations $\ell$-adiques, in Motives, AMS Proc. Symp. Pure Math. 55 (1994), vol. I, $377-400$.

[Se5] J.-P. Serre, Lectures on $N_{X}(p)$, A.K. Peters, 2012.

[T] S.G. Tankeev, On algebraic cycles on abelian varieties, II, Izv. Akad. Nauk. SSSR 43 (1979), 418-429.

Department of Mathematics and Computer Science, Adam Mickiewicz University, Poznań 61614, Poland

E-mail address: banaszak@amu.edu.pl

Department of Mathematics, University of California, San Diego, la Jolla, CA 92093, USA

E-mail address: kedlaya@ucsd.edu 